\title{
Investigation of the Effect of Local Buckling and VIV Fatigue on Failure Probability of Subsea Pipelines in Iranian South Pars Gas Field
}

\author{
Abdolrahim Taheri ${ }^{1 *}$, Mohammad Mahdi Shabani ${ }^{2}$, Mohammad Daghigh ${ }^{3}$ \\ ${ }^{1}$ Assistant Professor of Offshore Structural Engineering Department, Petroleum University of Technology (PUT); \\ Rahim.taheri@put.ac.ir \\ 2 MS.C. Student in Offshore Structural Engineering, Petroleum University of Technology (PUT); \\ m.shabani@mnc.put.ac.ir \\ ${ }^{3}$ Assistant Professor in Offshore Engineering, Pars Oil and Gas Company (POGC); mdaghigh@pogc.ir
}

\section{ARTICLE INFO}

Article History:

Received: 5 Aug. 2017

Accepted: 8 Jan. 2018

\section{Keywords:} subsea pipeline probability of failure free span local buckling

\begin{abstract}
Free-span occurs normally in a pipeline at uneven seabed, dynamic seabed and pipeline crossing. Free spanning in pipeline causes Vortex Induced Vibration (VIV) fatigue, fracture and bursting. In this paper, a pipeline located in South Pars Gas Field is assessed against local buckling and VIV fatigue using probability of failure theory based on the recommended methodology by Det Norske Veritas (DNV) corresponding to different soil classes and different span length to pipeline diameter and also different water depths by applying First-Order Reliability Method (FORM) and Monte-Carlo Sampling (MCS), separately. Furthermore, the simultaneous effect of local buckling and VIV fatigue is assessed in terms of probability of failure. Finally, in order to determine the effect of each parameter on failure probability, sensitivity analysis is carried out using the alpha index.
\end{abstract}

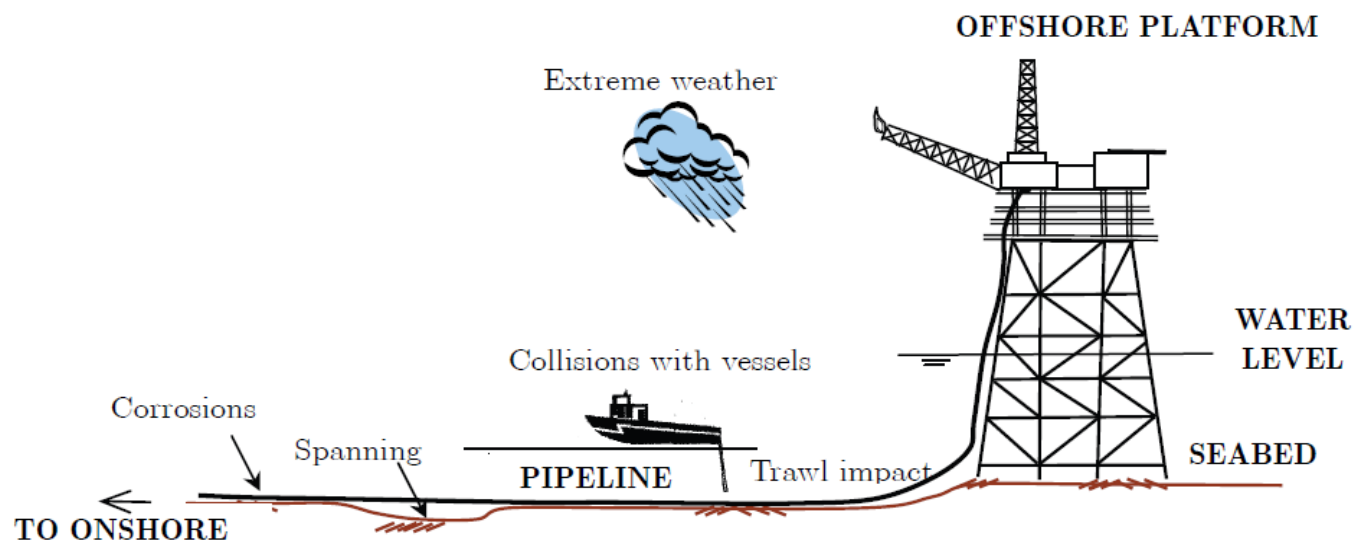

Figure 1. Different types of pipeline hazards[2]

\section{Introduction}

Marine pipelines, a complex system comprises a total length of thousands of kilometers, have been the most practical and low price means of transporting hydrocarbon including oil, gas, condensate and their mixtures in the offshore oil and gas industry[1]. The subsea pipelines are exposed to hazards like extreme weather conditions, collision with vessels, trawl impact and pipeline span (see Figure1)[2].

Subsea pipelines are subjected to various types of phenomena, like fatigue, corrosion, etc. which cause pipeline failure and should be monitored to guarantee the safety of pipeline [2]. According to DNV-OS-F101, fatigue assessment of pipeline must be performed at any stages (i.e. installation and operation)[3]. The free spanning as one of the important causes of fatigue occurs due to seabed unevenness, changes in seabed topology, artificial supports and scours [1], [4]. Furthermore, as water depths increases, deteriorative effect of local buckling on the pipeline health and integrity of offshore pipeline highlights. However, DNV recommends 


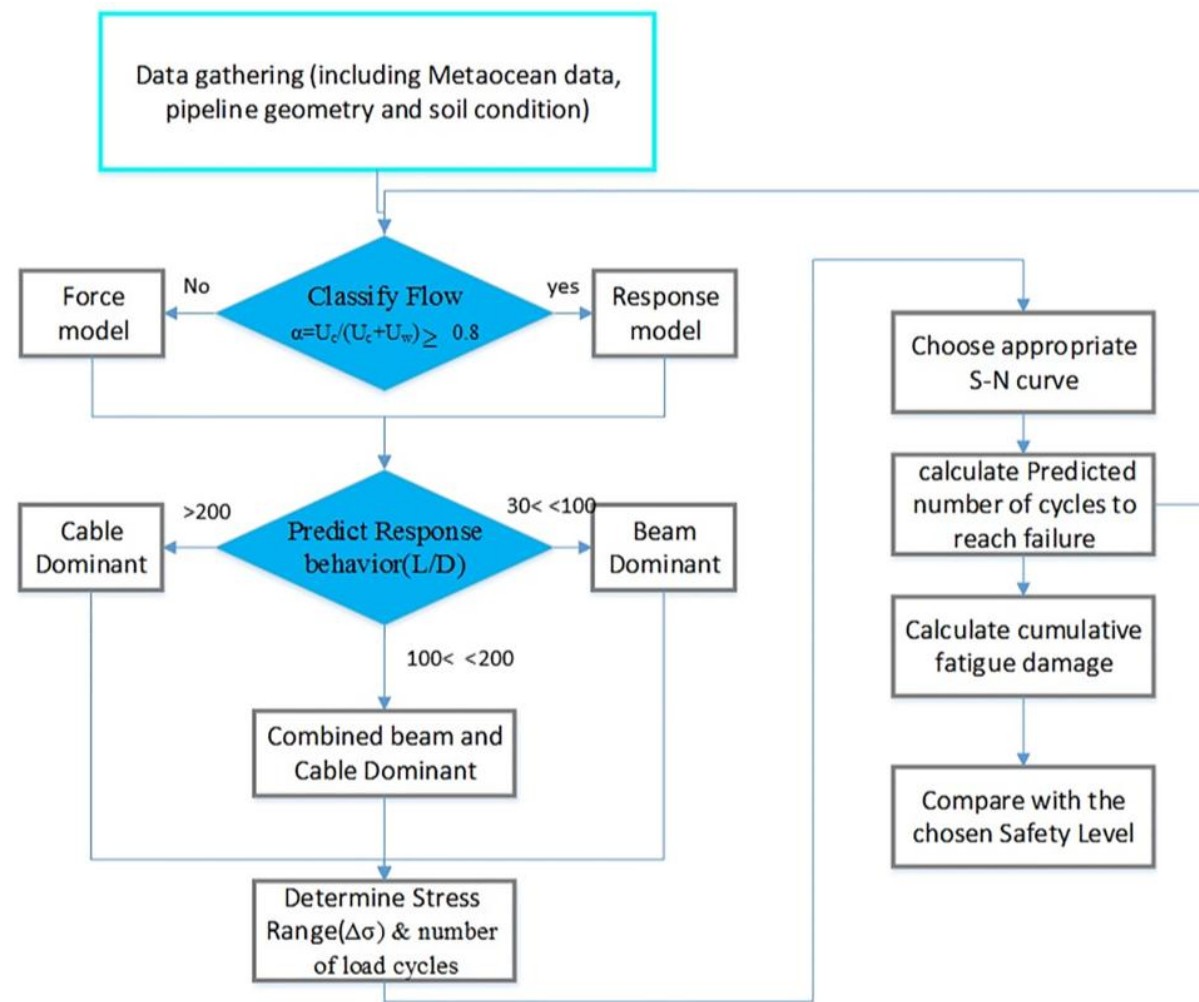

Figure 2. VIV fatigue assessment procedure[1,4,5]

some methodologies for assessing health of a submarine pipeline against local buckling and VIV fatigue separately, but in previous studies like Hagen[5], Abeele[6] and Shabani[7], simultaneous effect of VIV fatigue and local buckling was not considered.

In this paper, Probability of Failure (POF) of a pipeline based on DNV's recommended methodology, considering local buckling (considering combined load scenario) and free spanning using FORM and MCS is determined separately for a pipeline located in Iranian South Pars Gas Field. Furthermore, accuracy of FORM is tested in different conditions. As in previous studies, simultaneous effect of local buckling and VIV fatigue was not considered, therefore POF of the pipeline considering both conditions are determined. Finally, in order to assess the effect of each parameter on POF, sensitivity analysis is carried out using alpha index.

\section{Pipeline analysis}

\subsection{Free span}

The vortex shedding frequency caused by a flow normal to a free span is governed by the pipeline outer diameter, the current velocity, and the Strouhal's number. Once the shedding frequency reaches the natural frequency of a span, it starts to vibrate and VIV occurs[4]. The free span may induce the pipeline vibration due to vortex shedding which may eventually cause pipeline fatigue damage. Therefore, free span analysis is quite a fundamental aspect of the subsea pipeline design and operation. Because free span length limitation and fatigue damage are usually intervention due to the deep water depth and great seabed instability/unevenness[8].

In order to determine fatigue life capacity of a pipeline based on DNV code, following procedure should be followed (see Figure 2). In Figure 2, L is the span length, D is pipeline outside diameter (considering coating layer), $U_{c}$ is current velocity amplitude and $U_{w}$ is the significant wave-induced velocity amplitude.

DNV divided free spanning pipeline behavior into three categories based on ratio of span length to pipeline diameter; beam dominant behavior (for $r \cdot<\mathrm{L} / \mathrm{D}<100$ ), combined beam and cable behavior (for $100<\mathrm{L} / \mathrm{D}<\mathrm{r} \cdot$ ) ) and cable dominant behavior (for $\mathrm{L} / \mathrm{D}>\mathrm{r} \cdot \cdot)[8]$. In the first category, pipeline response can be estimated by deterministic theories, i.e. Bernoulli's beam theory. However, in second and third classes beam theory is not applicable and dynamic response must be predicted by solving equation of motion (more detailed information is given in reference [9]).

In order to perform VIV fatigue assessment, it is necessary to choose an appropriate fatigue criterion. The most popular fatigue criteria are displayed in Figure 3. 


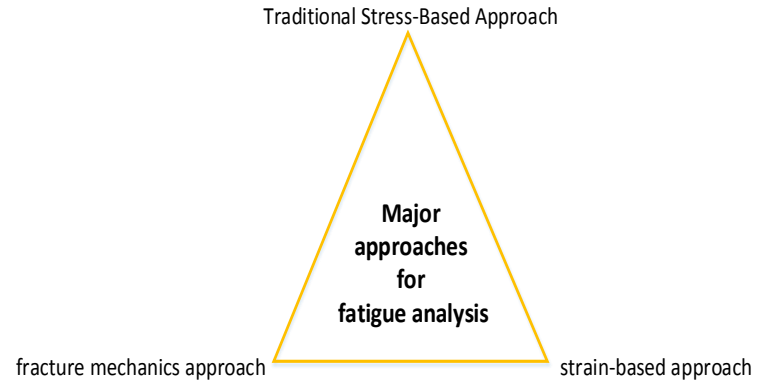

Figure 3. Major approaches for analyzing and designing fatigue[11]

DNV recommends application of stress-based approach [8], [12]. Main method for determining fatigue damage in stress-based approach is S-N curve [13]. Because of dependency of results on soil stiffness, soil characteristics should be determined using recorded data from site. In case of insufficient detailed information about seabed characteristics, DNV recommended to consider following models for calculating soil stiffness [8]:

$$
\begin{aligned}
K_{V} & =\frac{C_{v}}{1-v}\left(\frac{2}{3} \frac{\rho_{s}}{\rho}+\frac{1}{3}\right) \sqrt{D} \\
K_{L} & =C_{L}(1+v)\left(\frac{2}{3} \frac{\rho_{s}}{\rho}+\frac{1}{3}\right) \sqrt{D}
\end{aligned}
$$

where $K_{V}$ and $K_{L}$ are vertical and horizontal stiffness, $C_{v}$ and $C_{L}$ are dynamic stiffness factors in vertical and horizontal directions, respectively. $v$ is Poisson's ratio, $\rho_{s}$ is soil density and $\rho$ is water density. The value of the above parameters can be determined from DNV-RP-F105[8], [14].

\subsection{Local buckling}

Main load effect on subsea pipelines is bending combined with longitudinal force while subjected to external hydrostatic pressure during installation and internal pressure in operational phase[16]. A pipe subjected to bending may fail due to local buckling, collapse, or fracture, but it is local buckling or collapse limit state that commonly dictates the design. Local buckling and collapse strength of metallic pipes have been the main subjects for many studies in subsea and civil engineering, such as Murphey and Langner[17], Gresnigt[18], Mohareb et al.[19], and Bai et al.[20], [21].

The limit bending moment for steel pipes depends on many parameters. The major factors are given here in arbitrary sequence:

1. Diameter over wall thickness ratio.

2. Material stress-strain relationship.

3. Material imperfections.

4. Welding

5. Initial out-of-roundness.

6. Reduction in wall thickness

7. Cracks (in pipe or welding).
8. Local stress concentrations due to, say, coating.

9. Additional loads and their amplitude.

10. Temperature

Several formulations have been proposed for estimating collapse pressure like Timoshenko and Gere's[22], Haagsma and Schaap's[23] and etc. [3], [16], [24]. Both Timoshenko and Haagsma models account for initial out of roundness[16]. DNV-OSF101 uses Timoshenko and Gere's equation. DNV proposed two different conditions for pipeline collapse; overcoming external pressure as dominant factor and considering the combined effect of internal pressure, external pressure and axial force[3]. In order to determine collapse pressure due to combined loading effect, DNV proposed the following model;

$$
\begin{aligned}
& \left\{\begin{array}{l}
\left.\gamma_{m} \cdot \gamma_{S C} \cdot \frac{\left|\mathrm{M}_{S d}\right|}{\alpha_{c} \cdot M_{P}\left(t_{2}\right)}+\right\}^{2} \\
\left\{\frac{\gamma_{m} \cdot \gamma_{S C} S_{S d}\left(p_{i}\right)}{\alpha_{c} S_{P}\left(t_{2}\right)}\right\}^{2}
\end{array}\right\}^{2}+ \\
& \left(\alpha_{P} \cdot \frac{p_{i}-p_{e}}{\alpha_{c} \cdot P_{b}\left(t_{2}\right)}\right)^{\leq} \leq 1
\end{aligned}
$$

Where $\mathrm{M}_{S d}$ is the design moment (which can be determined by Eq.(4)), $S_{S d}$ is design axial force (which can be determined by Eq.(5)), $p_{i}$ is internal pressure, $P_{b}$ is bursting pressure (which can be found out by Eq.(6)), $S_{P}$ and $M_{P}$ are plastic capacity of pipeline against axial force and bending moment (which can be determined by Equations (7) and (8), respectively), $p_{e}$ is external pressure, $\gamma_{m}$ and $\gamma_{S C}$ are material resistance factor and safety class resistance factor, respectively, $\alpha_{c}$ and $\alpha_{P}$ are flow stress parameter and parameter for accounting the effect of $\mathrm{D} / \mathrm{t}_{2}$ ratio (which can be determined by Equations (9) and (10), respectively).

$M_{s d}=M_{F} \cdot \gamma_{F} \cdot \gamma_{c}+$

$M_{E} \cdot \gamma_{E} \cdot \gamma_{E}+$

$M_{I} \cdot \gamma_{F} \cdot \gamma_{c}+$

$M_{A} \cdot \gamma_{A} \cdot \gamma_{c}$

$S_{s d}=S_{F} \cdot \gamma_{F} \cdot \gamma_{c}+$

$S_{E} \cdot \gamma_{E}+S_{I} \cdot \gamma_{F} \cdot \gamma_{c}$

$+S_{A} \cdot \gamma_{A} \cdot \gamma_{c}+S_{A} \cdot \gamma_{A} \cdot \gamma_{c}$

$p_{b}(t)=\frac{2 t}{D-t} \cdot f_{c b} \cdot \frac{2}{\sqrt{3}}$ 
$S_{p}(t)=f_{y} \cdot \pi \cdot(D-t) t$

$M_{p}(t)=f_{y} \cdot(D-t)^{2} \cdot t$

$\alpha_{c}=(1-\beta)+\beta \cdot \frac{f_{u}}{f_{y}}$

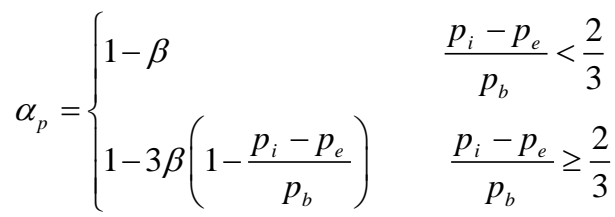

And in the Eq. (10), $\beta$ is a parameter for considering effect of $\mathrm{D} / \mathrm{t}_{2}$ which can be determined by Eq. (11)

[3].

$\beta=\frac{60-D / t_{2}}{90}$

Also in Eq. (4) and Eq. (5) $\gamma_{C}, \gamma_{F}, \gamma_{E}$ and $\gamma_{A}$ are condition load effect factor, functional load effect, environmental load effect and accidental load effect, respectively. As the contractor company (i.e. TOTAL Company) considered combined loading condition for assessment of pipeline against local buckling, current paper deals with combined loading condition.

\section{Reliability assessment}

\subsection{Reliability method}

Reliability of a component can be defined as the probability that it meets some specified requirements under special environmental conditions [25]. Reliability methods as a mathematical tool, are used for determination of POF in some special conditions by considering uncertainties in both load and resistance parameters [6]. Uncertainties can be divided into two main categories including epistemic and aleatoric[26]. Epistemic type is related to the measurement errors, limited sample numbers, or calibration of equipment, while aleatoric type is related to the nature of material or nature of phenomena. Aleatoric uncertainties, unlike the epistemic uncertainties, cannot be excluded by increasing the number of samples or calibrating the measurement's tools [27].

System reliability is defined as the probability that the system will not attain the specified limit state. Generally, performance function or Limit State Function (LSF) $g(X)$ is defined by the stochastic loads $L(X)$ and resistance $R(X)$ as the condition where load equals (or bigger than) the system's resistance:

$g(X)=R(X)-L(X)$

Mathematically, it is more suitable to calculate the reliability of a system in terms of its complements [6], [28]

$$
P_{f}=P(g \leq 0)=\int_{g \leq 0} f_{R}(x) f_{L}(x) d x
$$

Where $f_{R}$ and $f_{L}$ are probability density functions for resistance and load, respectively. Reliability index (RI) is defined as:

$$
\beta=-\phi^{-1}\left(P_{f}\right)
$$

where $\Phi$ is standard normal (cumulative) distribution function. POF and RI can be determined by FORM and MCS[29], [30].

\subsection{Case study}

In present study, reliability assessment is carried out for a pipeline located on South Pars Gas Field with the specifications presented as follows:

\begin{tabular}{|c|c|c|}
\hline Parameter & value & Unit \\
\hline Pipe class & API-5L-X65 & \\
\hline $\begin{array}{ll}\text { Pipeline } & \text { Wall } \\
\text { Thickness(PWT) } & \\
\end{array}$ & 24 & $\mathrm{Mm}$ \\
\hline Pipeline outside diameter & 816.8 & $\mathrm{Mm}$ \\
\hline Fluid type & \multicolumn{2}{|c|}{ Natural Gas } \\
\hline Fluid density & 110 & $\mathrm{Kg} / \mathrm{m}^{3}$ \\
\hline Steel density & 7850 & $\mathrm{Kg} / \mathrm{m}^{3}$ \\
\hline Water depth(maximum) & 85 & $\mathrm{M}$ \\
\hline Elasticity Modulus & 210 & $\mathrm{GPa}$ \\
\hline SMYS & 448 & $\mathrm{MPa}$ \\
\hline SMTS & 540 & $\mathrm{MPa}$ \\
\hline Operating Pressure $(\mathrm{OP})$ & 13.5 & $\mathrm{MPa}$ \\
\hline Ovality & $0.5 \%$ & \\
\hline $\begin{array}{l}\text { Submerged weight in } \\
\text { operating condition }\end{array}$ & 4273 & $\mathrm{~N} / \mathrm{m}$ \\
\hline Production & $\mathrm{We}$ & \\
\hline
\end{tabular}

Table 1. Pipeline specification

Environmental condition of pipeline installation site is approximately stable and its variation is small. Current speed varies from $0.5^{\mathrm{m} / \mathrm{sec}}$ to $0.6^{\mathrm{m} / \mathrm{sec}}$ in the worst condition (with one year and 100 years return period, respectively) and also effect of wave-induced flow on the oscillation amplitude is small and it can be neglected.

Pipeline seabed's profile is presented as follows:

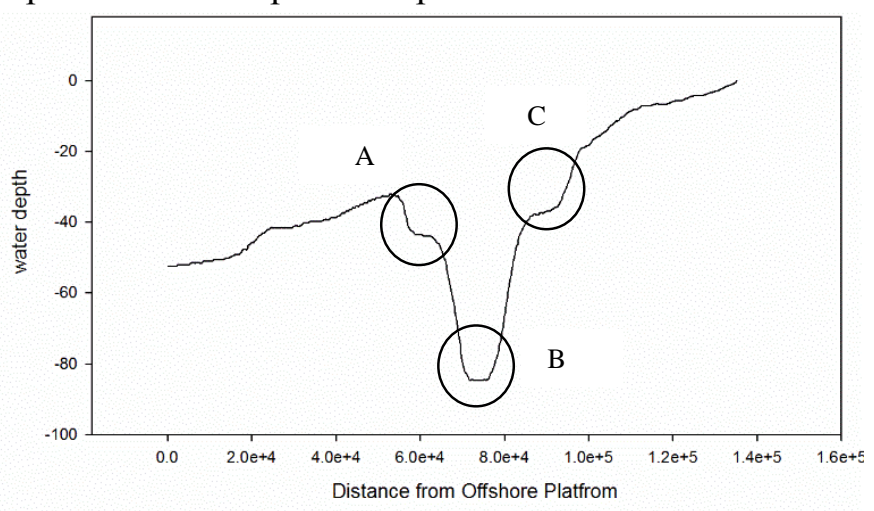

Figure 4. Seabed profile 
It can be found from Figure 4 that variation in slope of seabed is too much and in some points, (e.g. A) there are spans where free spanning may occur. However, designer company has not considered effect of VIV fatigue for the pipeline which is probable using above seabed profile for the pipeline. The present study assesses probability of occurrence of VIV fatigue for the pipeline.

\subsection{Target safety}

When the structural reliability analysis is needed to be carried out, a target safety level should be selected in order to ensure that a certain safety level is always achieved. For different probable scenarios, DNV-OSF101 recommends suitable target safety levels which are presented in Table 2. As free spanning belongs to the FLS and ULS categories and the designer was considered safety level as high class. Therefore, the target POF for satisfying the target safety level is adopted as $\mathrm{POF}=10^{-5}$.

Table 2. target POFs vs. target safety levels[3]

\begin{tabular}{llcccc}
\hline & \multicolumn{5}{c}{ Safety class } \\
& Limit state & & & \\
& & & & \\
ALS & all & $10^{-2}$ & $10^{-3}$ & $10^{-3}$ & $10^{-4}$ \\
\hline ULS & Pressure & $10^{-4}$ & $10^{-5}$ & $10^{-6}$ & $10^{-7}$ \\
& containment & to & to & to & to \\
& & $10^{-5}$ & $10^{-6}$ & $10^{-7}$ & $10^{-8}$ \\
\hline ULS & All other & & & & \\
FLS & & & & & \\
ALS & & $10^{-3}$ & $10^{-4}$ & $10^{-5}$ & $10^{-6}$ \\
\hline
\end{tabular}

\subsection{Limit State Function}

\subsubsection{Free spanning}

A comprehensive reliability analysis for free spanning subsea pipelines is presented in reference [5]. In this paper, in-line VIV fatigue is performed. Limit State Function (LSF) is used for fatigue failure after $T$ years which can be expressed as follows;

$g(T)=\left(T_{\text {fat }}(T) / 86400 * 365\right)-25$

Where $T_{\text {fat }}$ is the pipeline fatigue life capacity which can be determined by Eq.(16) and $\eta$ is usage factor which can be determined using DNV-RP-C203 and $D_{\text {fat }}^{\text {cum }}$ is cumulative fatigue damage that can be calculated by Eq. (17) [8], [12]. However DNV recommends that pipeline design life should be considered at least for 25 years [2], [3].

$T_{\text {fat }}(T)=\eta / D_{\text {fat }}^{\text {cum }}$
$D_{\text {fat }}^{\text {cum }}=\sum_{i=1}^{k} \frac{n_{i}}{N_{i}}$
In addition to the above LSF, the Palmer-Miner index can be used to develop another LSF for reliability assessment of free spanning pipelines (see Eq. (18)) [6].

$g(x)=1-D_{\text {fat }}^{\text {cum }}$

\subsubsection{Local buckling}

In order to choose an appropriate LSF for local buckling, using DNV's recommended equation for assessment of pipeline collapse under combined loading (Eq. (3)) and TOTAL's design documentation, the following LSF is chosen for reliability assessment of pipeline collapse under combined loading.

$g=1-\left\{\begin{array}{l}\gamma_{m} \cdot \gamma_{S C} \cdot \frac{\left|\mathbf{M}_{S d}\right|}{\alpha_{c} \cdot M_{P}\left(t_{2}\right)}+ \\ \left\{\frac{\gamma_{m} \cdot \gamma_{S C} S_{S d}\left(p_{i}\right)}{\alpha_{c} S_{P}\left(t_{2}\right)}\right\}^{2}\end{array}\right\}^{2}-$

$\left(\alpha_{P} \cdot \frac{p_{i}-p_{e}}{\alpha_{c} \cdot P_{b}\left(t_{2}\right)}\right)^{2}$

For positive values of $g$, pipeline can continue a safe operation, and in other situations, pipeline failure will occur.

\subsection{Uncertainties}

Uncertainties which are considered for reliability assessment are described in Table 3 with their relevant mean value and Coefficient of Variation (C.O.V). Distribution types and their relevant parameters are mentioned in Table 3 which and are based on recommendation of following references [6], [25].

Table 3. Uncertainties of parameters with their relevant mean and C.O.V

\begin{tabular}{ccccc}
\hline Row & Parameter & $\begin{array}{c}\text { Distribution } \\
\text { Type }\end{array}$ & Mean & C.O.V \\
\hline 1 & PWT & Normal & 0.024 & 0.05 \\
\hline 2 & $\begin{array}{c}\text { Concrete } \\
\text { coating layer }\end{array}$ & Normal & 0.05 & 0.1 \\
\hline 3 & Pipeline span & Weibull & Variable & Variable \\
\hline 4 & $\begin{array}{c}\text { Pipeline } \\
\text { diameter }\end{array}$ & Normal & 0.8168 & 0.05 \\
\hline 5 & $\begin{array}{c}\text { Young's } \\
\text { modulus }\end{array}$ & Log-normal & $210 \times 10^{9}$ & 0.05 \\
\hline 6 & $\begin{array}{c}\text { S-N curve } \\
\text { scaling } \\
\text { parameter }\end{array}$ & Normal & 1 & 0.3 \\
\hline 7 & Soil stiffness & Normal & Variable & Variable \\
\hline 8 & Water depth & Weibull & Variable & Variable \\
\hline 9 & $\begin{array}{c}\text { Internal } \\
\text { pressure }\end{array}$ & Normal & $13.5 \times 10^{6}$ & 0.1 \\
\hline 10 & $\begin{array}{c}\text { S-N curve } \\
\text { scaling } \\
\text { parameter }\end{array}$ & Normal & 1 & 0.3 \\
\hline 11 & Fy & Normal & $448 \times 10^{6}$ & 0.1 \\
\hline 12 & Fu & Normal & $540 \times 10^{6}$ & 0.1 \\
\hline
\end{tabular}




\section{Result and discussion}

In order to assess the pipeline integrity against mentioned failure modes, safety of pipeline against each failure modes are assessed separately. Afterwards, simultaneous effect of local buckling and free spanning is considered and pipeline safety is assessed in terms of POF. Results of each failure modes and their relevant consequences are presented and discussed in the following sections;

\subsection{VIV fatigue}

Using the recommended LSF (Eq. (15)) and considering the presented uncertainties in Table 3 and also using the pipeline specifications mentioned in Table 1, POF is determined with respect to six clay classes and three sand types and also different L/D ratios. Table $5 \&$ Table 6 show the relationship between $\mathrm{POF}$ and L/D for different seabed clay and sand types.

Table 4. POF for different $L / D$ and clay types

\begin{tabular}{ccccccc}
\hline \multicolumn{7}{c}{ Response dominated by combined beam and cable } \\
behavior
\end{tabular}

It is obvious from Table 4 that no failure will occur in beam dominant response behavior $(30<\mathrm{L} / \mathrm{D}<100)$ and for very soft and soft clay classes. For larger L/D, FORM's results approach to the MCS's results; therefore, FORM analysis can be applicable for larger ratios of span length to pipeline diameter. Furthermore, it is noted that POF increases by improving soil stiffness and POFs of very stiff and hard clays soils are approximately similar. Also, variation of POF with respect to different span length to pipeline diameters and different classes of clay is shown in the following Figure;

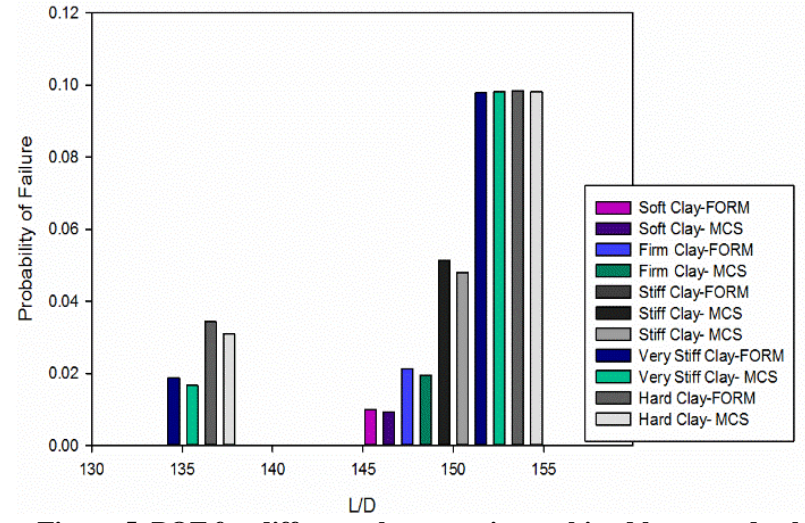

Figure 5. POF for different clay types in combined beam and cable response behavior domain

Table 5. POF for different $L / D$ and sand types

\begin{tabular}{|c|c|c|c|}
\hline \multirow{2}{*}{ L/D } & \multicolumn{3}{|c|}{$\begin{array}{l}\text { Response dominated by combined beam and } \\
\text { cable behavior }\end{array}$} \\
\hline & 110 & 120 & 150 \\
\hline
\end{tabular}

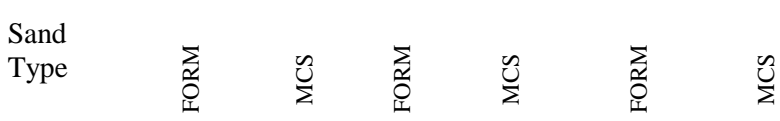

\begin{tabular}{ccccccc}
\hline Loose & $1.1 \mathrm{e}-16$ & 0 & 0.025 & 0.021 & 0.053 & 0.050 \\
\hline Medium & $5.1-14$ & 0 & 0.018 & 0.016 & 0.082 & 0.080 \\
\hline Hard & $1.3 \mathrm{e}-10$ & 0 & 0.056 & 0.054 & 0.095 & 0.096 \\
\hline
\end{tabular}

It can be found from Table 5 that in comparison with target safety POF, there is no failure for lower L/D ratios. Also differences between FORM's result and MCS's result are low in larger ratio of span length to pipeline diameter. Besides in case of stiffer sands difference between FORM's result and MCS's result tends to the lowest value. Therefore, FORM can be applied for the larger span length to pipeline diameter and its accuracy increases for stiffer sands. Furthermore, it is noted from Table 5 that for larger span length to pipeline diameter, POF increases. Also, variation of POF with respect to different span length to pipeline diameter ratios and different types of sand is displayed in the following Figure;

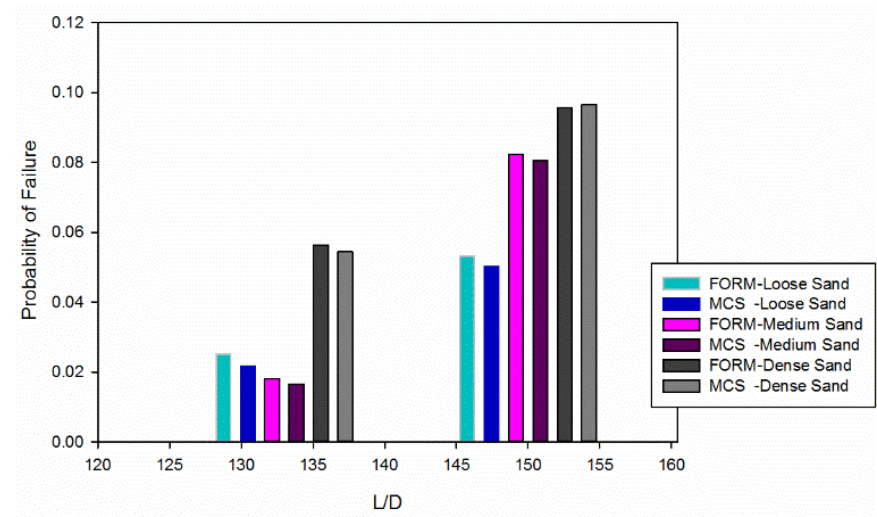

Figure 6. Variation of POF vs. different L/D

Furthermore, changes in importance of each parameter on POF, regarding different L/D and clay types are investigated in detail in Figure 7 \& Figure 8; 

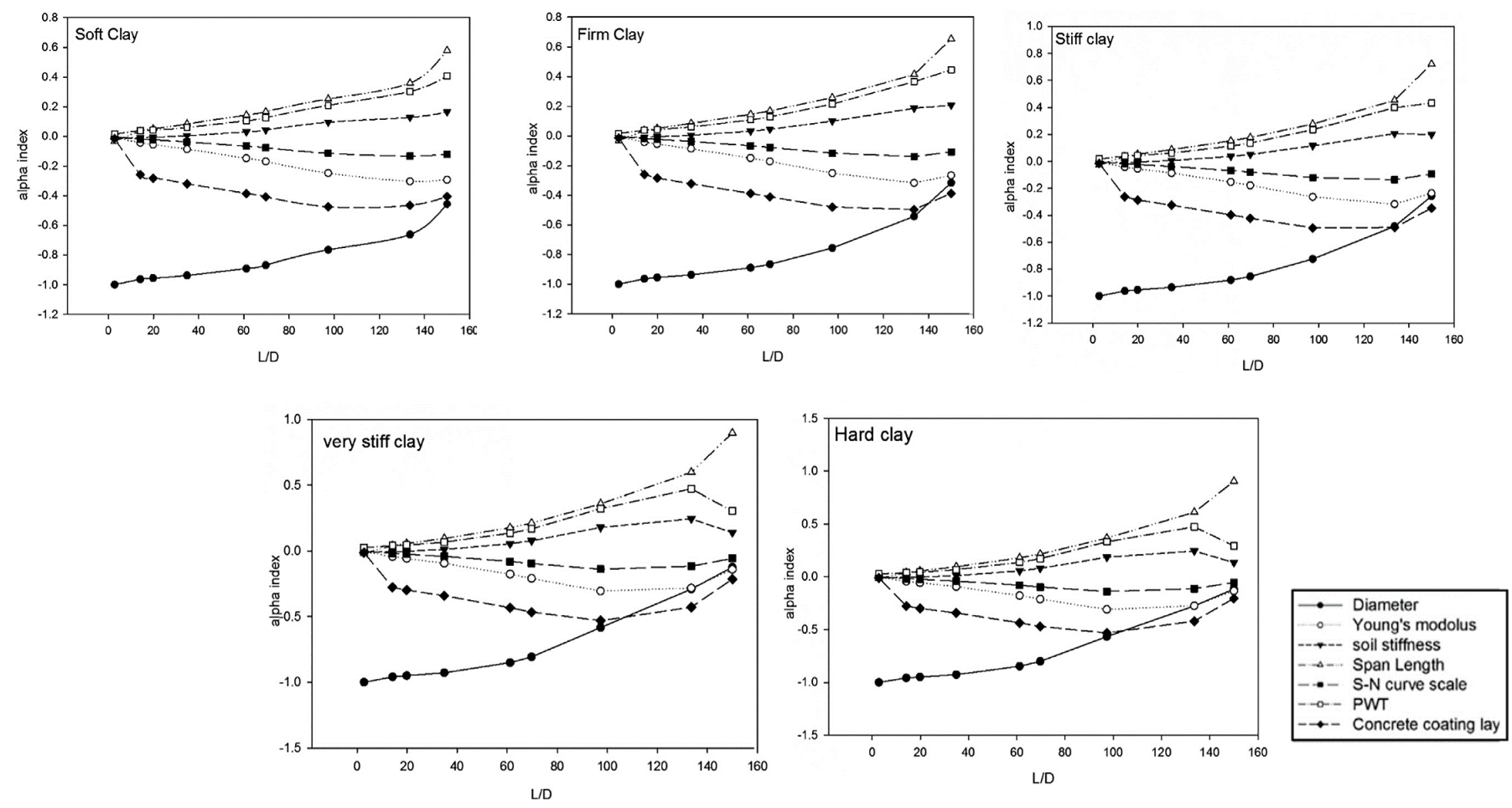

Figure 7. Variation of alpha index vs. L/D for sandy soil
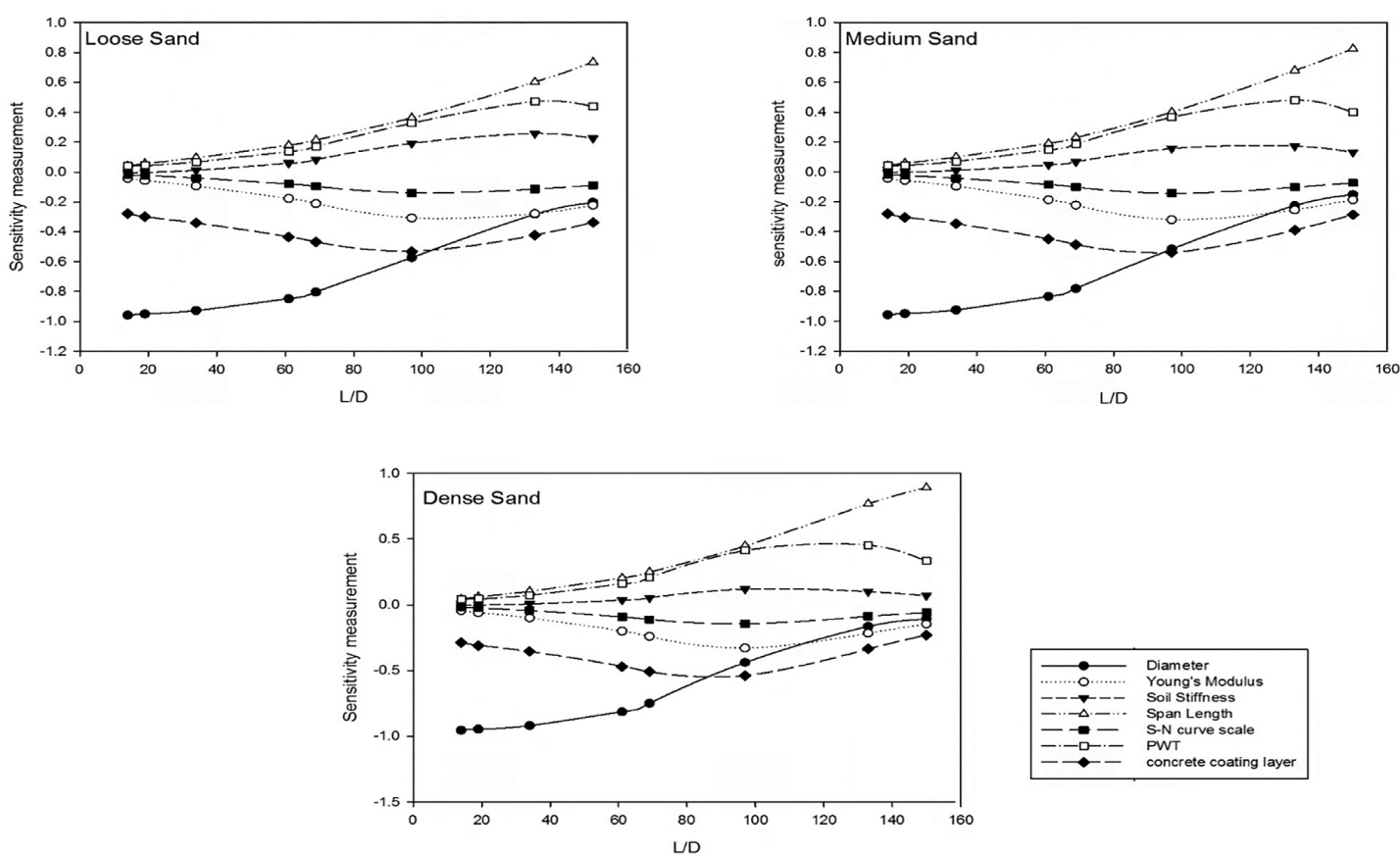

Figure 8. Variation of POF vs. L/D for sand seabed

As shown in Figures 7 and 8, except S-N scaling parameter, variation of other parameters influences the alpha index for different L/D. By performing sensitivity analysis for different $\mathrm{L} / \mathrm{D}$, decision makers will find the right choice to decide which parameter can provide more resistance for pipeline and by considering the economic aspects, choose the best option for repair or even replace.

\subsection{Local buckling}

Using recommended LSF (i.e. Eq. (19)) and considering the presented uncertainties in Table 3 and also using the pipeline specification mentioned in Table 1, POF is determined corresponding different water depths. Relationship between POF and different water depths is shown in the Figure 9; 


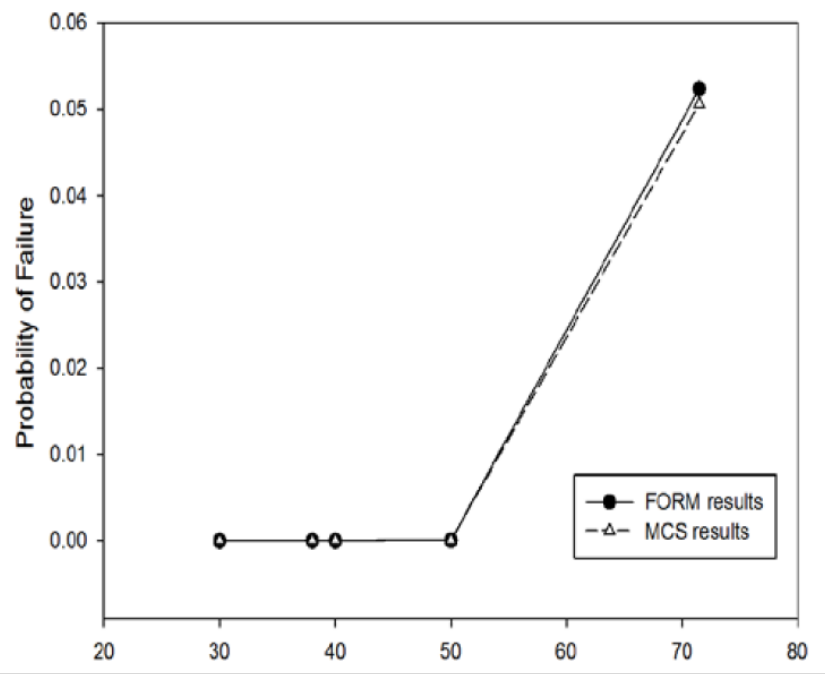

Figure 9. POF of the pipeline vs. different water depth

It can be found from figure 9, that no failure will not happen for water depth lower than 45 meters. POF for deeper waters goes up to five percent. Furthermore, it is noted from Figure 5 that FORM's results are close to MCS's results (considering 10,000,000 samples).

Therefore, FORM can be applied for assessment of the pipeline against local buckling.

Furthermore, in order to assess effects of each parameter on POF (using alpha-index) sensitivity analysis is carried out which its result is presented in following;

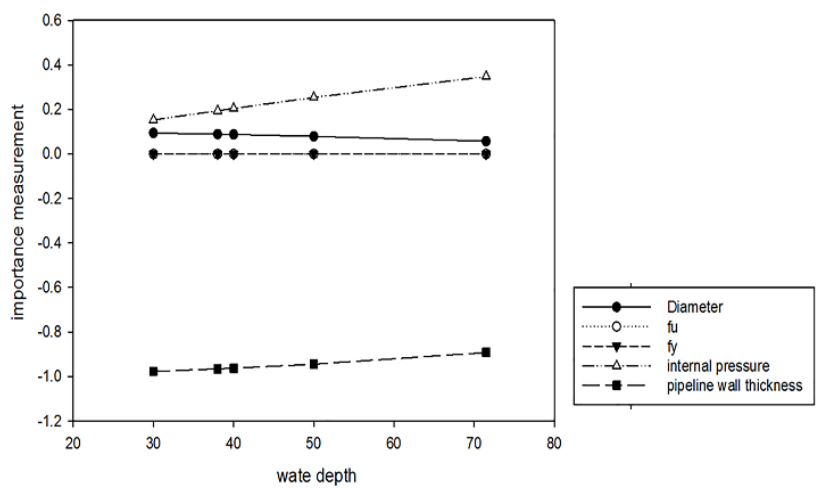

Figure 10. Variation value of importance measurement vs. water depth

As shown in Figure 10, according to the positive and negative values of importance measurement, PWT and pipeline diameter are the most effective capacity and load parameters, respectively. Furthermore, strength parameters of pipeline remain constant for different water depths.

\subsection{Combined effect of VIV fatigue and local buckling}

In order to assess health and integrity of pipeline against combined effect of local buckling and VIV fatigue, there has been no serious attempt to present a formulation, there is no successful attempts like[31] and etc. In some special researches like galgoul[31] it is shown that VIV fatigue has no serious effect on buckling behavior of pipeline. Therefore, in this research free spanning and local buckling are proposed independent mathematically. According to the set theory, simultaneous POF of two outcomes can be determined as follow

$P(A \cap B)=P(A) * P(B)$

erefore, the joint $\mathrm{POF}$ of the pipeline against combined effect of free spanning and local buckling equals to POF of VIV fatigue times POF of local buckling. Result of the assessment against combined effect of VIV fatigue and local buckling is presented in following;

Table 6. POF of the pipeline for interacted outcomes considering different clay classes

\begin{tabular}{lcccc}
\hline L/D & \multicolumn{2}{c}{120} & \multicolumn{2}{c}{150} \\
\hline $\begin{array}{l}\text { Clay } \\
\text { Type }\end{array}$ & $\sum^{2}$ & $\sum_{1}$ & $\sum_{0}$ & $\sum^{2}$ \\
\hline $\begin{array}{c}\text { Very Soft } \\
\text { Clay }\end{array}$ & 0.0 & 0.0 & 0.0 & 0.0 \\
\hline Soft Clay & 0.0 & 0.0 & 0.0 & 0.0 \\
\hline Firm Clay & 0.0 & 0.0 & $1.01 \mathrm{E}-10$ & $1.01 \mathrm{E}-10$ \\
\hline $\begin{array}{c}\text { Stiff Clay } \\
\text { Very Stiff } \\
\text { Clay }\end{array}$ & $1.40 \mathrm{E}-08$ & $1.41 \mathrm{E}-08$ & $2.69 \mathrm{E}-03$ & $2.25 \mathrm{E}-03$ \\
\hline Hard Clay & $1.84 \mathrm{E}-04$ & $1.02 \mathrm{E}-03$ & $5.12 \mathrm{E}-03$ & $5.53 \mathrm{E}-03$ \\
\hline
\end{tabular}

Table 7. POF of the pipeline for interacted outcomes considering different sand classes

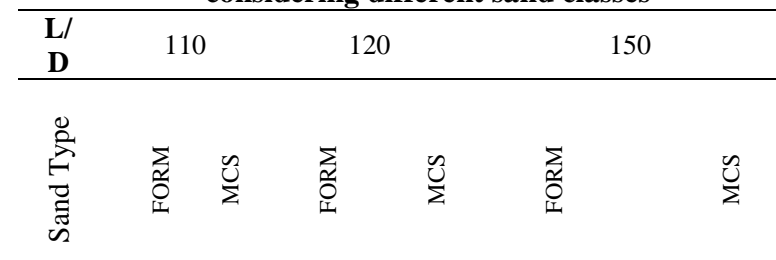

$\begin{array}{lllllll}0 & & & & & & \end{array}$

$\begin{array}{lllllll}\text { 爱 } & 0.0 & 1.02 \mathrm{E}-04 & 0.0 & 9.07 \mathrm{E}-04 & 4.29 \mathrm{E}-03 & 4.53 \mathrm{E}-03\end{array}$

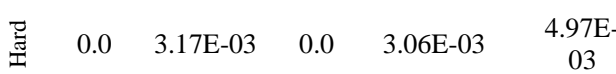

It can be found from Table 7 that no failure will occur for the shallow waters (i.e. $\mathrm{h}<71.5^{\mathrm{m}}$ ), and pipeline will continue a safe operation in those depths. Furthermore, in comparison with selected target safety POF, pipeline will remain in safe zone for soft clay soils and lower span length to pipeline diameter ratio than 120 . Another interesting point is that stiffening soil causes increase in POF due to combined effect of local buckling and free spanning. Besides FORM's result are 
again close to MCS's results for the stiffer clays and larger span length to pipeline diameter. Therefore, FORM can be applied for those conditions.

It can be understood from Table 7 that for values of span length to pipeline diameter larger than 110 meters, failure of pipeline would be considered as ineligible, therefore predicting devices like buckle arrestor should be used as means of buckling control [3]. Furthermore, like clay soils, increase in soil stiffness causes increase in POF. Another interesting point is that FORM can be applied for all categories of sands because of small difference between FORM's results and MCS's results $(10,000,000$ samples $)$.

\section{Conclusion}

In this paper, FORM and MCS were utilized for determining the reliability of a subsea pipeline located in South Pars Gas Field, which was exposed to free span (single span) and local buckling. Six classes of clay and three sand types, different water depth and different span length to pipeline diameter were considered to determine POF of the pipeline.

It is concluded that FORM can be applied for reliability assessment of spanning pipeline in case of larger span length to pipeline diameter and stiffer soil seabed. Furthermore, it can be used for reliability assessment of the pipeline against local buckling.

It is concluded that, in comparison with selected target safety POF, for soft soils like very soft clay and soft clay, no failure will occur. It is also concluded that pipeline collapse will not affect the integrity of pipeline and for deeper depths, some prevention measures like buckle arrestor should be taken.

Furthermore, in this research, free spanning and local buckling considered as independent phenomena. It is concluded that, however, in comparison with selected target safety POF and considering combined effect of VIV fatigue and local buckling, pipeline failure will occur; however, its relevant POF is too small which can be neglected.

Finally, sensitivity analysis using alpha index is carried out. It is shown that span length and pipeline diameter are the most effective load and capacity parameters, respectively.

It is strongly recommended to consider FE models for investigation accurate interaction between local buckling and VIV fatigue of pipeline in future works.

\section{References}

1- Bai, Q. and Bai, Y., (2014), "1 - Introduction," in Subsea Pipeline Design, Analysis, and Installation, Boston: Gulf Professional Publishing, p. 3-21. doi: 10.1016/B978-0-12-386888-6.00001-8

2- Mustaffa, Z., (2011), System Reliability Assessment of Offshore Pipelines, $\mathrm{PhD}$ Thesis, University of Delft, Netherland.

3- "DNV-OS-F101: Submarine Pipeline Systems (2010).
4- Rezazadeh, K., Zhu, L., Bai, Y., and Zhang, L. , (2010), Fatigue Analysis of Multi-Spanning Subsea Pipeline, In Proceedings of 29th International Conference on Ocean, Offshore and Arctic Engineering: Volume 5, Parts A and B, p. 805-812. doi: 10.1115/OMAE2010-20847

5- Hagen, O., Mork, K. , Sigurdsson, G. , and Nielsen, F. G., (2003), Evaluation of Free Spanning Pipeline Design in a Risk Based Perspective, Vol. 2 Safety Reliability, Pipeline Technology, vol. 2, p. 789-799,. doi: 10.1115/OMAE2003-37419

6- Van den Abeele, F. , Boël, F. , and Vanden Berghe, J.-F. , (2014), Structural Reliability of Free Spanning Pipelines, Volume 3: Materials and Joining; Risk and Reliability, doi: 10.1115/IPC2014-33552

7- Shabani, M. M. , Taheri, A., and Daghigh, M., (2017), Reliability Assessment of Free Spanning Subsea Pipeline, Thin-Walled Structures, vol. 120, p. 116-123, doi: 10.1016/j.tws.2017.08.026

8- "DNV-RP-F105: Free Spanning Pipelines, (2006).

9- Wilson, J. F., J.Muga, B., and C.Reese, L., (2003), Dynamics of Offshore Structrues, Second edition. Hoboken, New Jersey: John Wiley \& Sons, Inc.,

10- Van den Abeele, F. , Boël, F., and Hill, M., (2013), Fatigue Analysis of Free Spanning Pipelines Subjected to Vortex Induced Vibrations, In Proceedings of the 32rd International Conference on Ocean, Offshore and Arctic Engineering OMAE2013, doi: 10.1115/OMAE2013-10625

11- Dowling, N. E. , (2013), Fatigue of Materials: Introduction and Stress-Based Approach," in Mechanical Behavior of Materials: Engineering Methods for Deformation, Fracture, and Fatigue, Fourth edition, p. 416-490.

12- DNV GL, (2016 ), DNVGL-RP-C203: Fatigue Design of Offshore Steel Structures, no. DNVGL-RPC203.

13- Dowling, N. E. , Prasad, K. S., and Narayanasamy, R., (2013), Mechanical Behavior of Materials: Engineering Methods for Deformation, Fracture, and Fatigue, Editors: K. S. Prasad and R. Narayanasamy, Pearson, pp. 26-30.

14- Fyrileiv, O. , and Kim, M. , (1998), Assessment of Free Spanning Pipelines Using the DNV Guideline, In Proceeding of $8^{\text {th }}$ International Offshore Polar Engenergy Conference, vol. II, p. 100-106.

15- Sollund, H. A. and Vedeld, K., (2015), Effects of Seabed Topography on Modal Analyses of Free Spanning Pipelines, In Proceeding of International Offshore Polar Engergy Conference, pp. 106-114, 2015.

16- Bai, Q. and Bai, Y., (2014 ), Chapter-3: Buckling and Collapse of Metallic Pipes, in Subsea Pipeline Design, Analysis, and Installation, editors: Q. Bai and Y. Bai, Boston: Gulf Professional Publishing, p. 41-65. doi: 10.1016/B978-0-12-397949-0.00003-0

17- Murphey, C. E., and Langner, C. G. , (1985), Ultimate Pipe Strength Under Bending, Collapse And 
Fatigue, In Proceedings of the $4^{\text {th }}$ International Conference on Offshore Mechanics and Arctic Engineering, vol. 1, p. 467-477.

18- Gresnigt, A. M. , (1987), Plastic Design of Buried Steel Pipelines in Settlement Areas.

19- Mohareb, M. E. , (1994), deformational Behaviour of Line Pipe, $\mathrm{PhD}$ Thesis, Unverisity of Albereta, Canada.

20- Bai, Y. , Tang, J. , Xu, W. , and Ruan, W. , (2015), Reliability-Based Design of Subsea Light Weight Pipeline Against Lateral Stability, Journal of Marine Structures, vol. 43, p. 107-124, doi: 10.1016/j.marstruc.2015.06.002

21- young, B. , Ranger, I. , and Torgeir, M. , Tube Collapse Under Combined Pressure, Tension And Bending Loads, International Journal of Offshore Polar Engineering, vol. 3, no. 2.

22- STEPHEN, T. , and JAMES, G. , (1963), Theory On Elastic Stability, New Yourk: Mcgraw-Hill Publication, p. 290-300.

23- Haagsma, S. C., and Schaap, D. , (1981), Collapse Resistance of Submarine Lines Studied, Oil Gas Journal, United States, vol. 79.

24- Bai, Y. , and Bai, Q., (2005), Chapter 3: Buckling/Collapse of Deepwater Metallic Pipes, in Subsea Pipelines and Risers, Editors: Y. Bai and Q. Bai, Oxford: Elsevier Science Ltd, p. 41-66.

25- Lee, O. S., Kim, D. H. , and Choi, S. S. , (2006) Reliability of Buried Pipeline Using a Theory of
Probability of Failure, Solid State Phenomena, vol. 110, p. 221-230.

26- BOMEL Limited, (2001), Probabilistic Methods: Uses and Abuses in Structural Integrity, in Probabilistic methods: Uses and abuses in structural integrity, no. 398/2001.

27- BOMEL Limited, Structural Reliability Theory, Uncertainty Modelling and the Interpretation of Probability, in Probabilistic methods: Uses and abuses in structural integrity, no. 398/2001, 2001.

28- Der Kiureghian, A., (2005), First- and SecondOrder Reliability Methods, in Engineering Design Reliability, Editors: E. Nikolaidis, D. M. Ghiocel, and S. Singhal, ohio: CRC Press, p. 302-325.

29- Mahmoodian, M. , and Li, C. Q. , (2017), Failure Assessment and Safe Life Prediction of Corroded Oil And Gas Pipelines, Journal of Petroleum Science Enginering, vol. 151, p. 434-438. doi: 10.1016/j.petrol.2016.12.029

30- Schuëller, G. I. , and Stix, R. , (1987), A Critical Appraisal of Methods to Determine Failure Probabilities, Structural Safety, vol. 4, no. 4, pp. 293 309. doi: 10.1016/0167-4730(87)90004-X

31- Galgoul, N. S. , Paulino de Barros, J. C. , and Ferreira, R. P., (2004), The Interaction of Free Span And Lateral Buckling Problems, In Proceedings of International Pipeline Conference, Volumes 1, 2, and 3, p. 1905-1910. doi: 10.1115/IPC2004-0308 